\title{
Representaciones sociales sobre ciudadanía en estudiantes de educación media técnico profesional: el caso de una escuela industrial salesiana de Santiago
}

\author{
Mag. Juan Carlos Toro Cornejo ${ }^{1}$
}

\begin{abstract}
RESUMEN
El presente artículo indaga en las representaciones sociales sobre ciudadanía que poseen los estudiantes de educación media técnico-profesional pertenecientes a una escuela industrial de Santiago. Estos sentidos resultan relevantes de conocer debido a que, durante las últimas décadas, los estudiantes secundarios chilenos han manifestado un alto desinterés por acceder a instancias de participación ciudadana, como consecuencia de la desarticulación que provocó la dictadura militar y las decisiones de los siguientes gobiernos en desmedro de reivindicaciones de tipo social. Mediante un enfoque de tipo cualitativo, planteado desde una perspectiva hermenéutica, con un diseño basado en un estudio de caso, utilizando la entrevista semiestructurada como técnica de recolección de información y una muestra de doce estudiantes de cuarto año medio de las distintas especialidades, se efectuó un análisis interpretativo del contenido recopilado. A partir de los significados que le otorgan dichos jóvenes al concepto de "ciudadanía", surgen elementos tales como sentidos de pertenencia territorial, aspectos de índole civil, política y social. Además, se relevan agentes como la familia, la escuela y la internet en la construcción de estas significaciones.
\end{abstract}

Palabras clave: Ciudadanía, representaciones sociales, educación media técnico-profesional.

\section{Social representations on citizenship in students of technical- professional high school: the case of a Salesian Industrial School of Santiago}

\section{ABSTRACT}

This article explores the social representations of students in technical-professional high school about citizenship, in Santiago. These senses are relevant to know, since during recent decades Chilean secondary students have expressed a high lack of interest related with accessing to the request of citizen participation, as a result of disarticulation caused by the military dictatorship and the decisions of following governments in detriment of

I Ingeniero en Electrónica. Profesor de Educación Media Técnico Profesional. Magíster en Educación mención Currículum e Innovaciones Pedagógicas. Profesor Especialista Curricular, Área Académica de Electricidad y Electrónica, Universidad Tecnológica de Chile INACAP. E-mail: juan.toro29@inacapmail.cl 
social demands. Using a qualitative approach behind a hermeneutic perspective, with a case-study design, utilizing the semi structured interview like an information collection technic, and a sample of twelve high school fourth-year students of different specialities, an interpretative analysis of the collected contents was made. From the meanings, which are given by these young people to the concept of citizenship, arise elements such as territorial belonging senses, civil, political and social aspects. In addition, agents like family, school and Internet are relieved in the construction of these meanings.

Keywords: Citizenship, social representations, technical-professional high school.

\section{Introducción}

La "ciudadanía", como una categoría política asociada a la pertenencia y la participación de personas dentro de una sociedad, ha adquirido en los últimos años una especial relevancia social y por sobre todo educativa, en la que se visualiza la necesidad de comprender la manera como se accede a la condición de ciudadano (Mata, Ballesteros y Padilla, 2013). Cuando la mirada se focaliza en la juventud, como parte del entramado social y en tránsito hacia la adquisición de derechos permanentes otorgados por el Estado, surge la interrogante sobre cómo este segmento se vincula con la ciudadanía y su ejercicio. Sandoval (2003) considera que el contexto actual crea una densa atmósfera en su entorno, a partir de factores tales como la globalización y todas las desigualdades que genera, las diversas formas de organizarse que poseen hoy los jóvenes, el descrédito experimentado por el poder político, que los hace restarse de aquel espacio, y los aspectos formales que reconocen a cierto número de ellos como ciudadanos de pleno derecho por su condición de mayoría de edad, entre otros grandes dilemas que los sitúan finalmente como sujetos peligrosos y transgresores, donde la noción de "ciudadanía" que opera al interior del conglomerado no representa lo mismo para todos.

Dentro de la sociedad chilena es posible apreciar que el fin de la dictadura en el año 1990 y el aumento en los índices de crecimiento del país elevaron en las décadas siguientes las condiciones de acceso a bienes de consumo y educación, evolucionando de manera dispar con relación al nivel social y de inclusión de todos quienes la conforman (Asún, Ruiz, Aceituno, Venegas y Reinoso, 2012), siendo una prueba de ello la inequidad en la distribución de los ingresos del 
país, la mayor entre los países pertenecientes a la Organización para la Cooperación y el Desarrollo Económico (OCDE). Por esto reviste importancia la construcción social de un proyecto país igualitario, equitativo e inclusivo, el cual requiere de personas con capacidad crítica y propositiva ante situaciones que vive la sociedad chilena, siendo un factor relevante la formación ciudadana recibida por los jóvenes en las instituciones escolares, en especial en aquellas pertenecientes al mundo de la educación secundaria con foco en la formación diferenciada técnico-profesional (TP), considerando que sus estudiantes conforman hoy cerca del $40 \%$ de la matrícula total de la enseñanza media en Chile (Larrañaga, Cabezas y Dussaillant, 20I4).

\section{Ciudadanía, juventud y educación}

Precisar el concepto de "ciudadanía" reviste la complejidad que encierra toda definición: la circunscripción fenomenológica, es decir, la limitación a una mirada en desmedro de las perspectivas posibles respecto de un fenómeno. Pese a ello, y partiendo de la base que "ciudadanía" es la abstracción que opera como continente de ciudadano, es menester dar cuenta de tal categoría desde la visión del sujeto. Así las cosas, puede afirmarse que "ciudadano" es el habitante de una comunidad, de un lugar enmarcado en el espacio sociotemporal; es el perteneciente y a quien pertenece el derecho a ser parte de una cultura en particular.

A partir de Aristóteles, el concepto de "ciudadano" es planteado desde la perspectiva del hombre libre que se forma en la ciudad, o sea el ciudadano es una construcción colectiva que solo es posible en la ciudad; este es un principio fundamental al que no se puede renunciar (Alejandre y Escobar, 2009, p. I06). Desde la sociología del conocimiento (Berger y Luckmann, 200I) puede ampliarse la afirmación anterior, en tanto que la realidad, como construcción, incluye la inicial consideración del sujeto implicado en lo real, de su dimensión social, su posibilidad de cuestionamiento y su sustrato ideológico.

Los conceptos de "ciudadano" y "ciudadanía" se circunscriben a campos semánticos distintos: uno relativo a la pertenencia y otro 
al comportamiento social. Lo anterior plantea la interrogante zes inherente al sujeto o se obtiene como consecuencia de acciones sociales? Luego, esto es, según Lizcano (2012, p. 7), parte de un conflicto mayor: la diferencia entre nacionalidad y ciudadanía. El autor plantea que la nacionalidad "es una condición de sometimiento político de una persona a un Estado determinado”, mientras que, ciudadanía es un atributo que se adquiere bajo ciertas condiciones de posesión de nacionalidad y cumplimiento de condiciones legales. De este modo, la necesidad de ciudadanía implicará la elaboración de un discurso que conduzca a tal calidad, que en términos genéricos se construye respecto de valores como: interacción democrática, participación cívica, igualdad jurídica, respeto por la pluralidad y la libertad, defensa de derechos individuales y reflexividad crítica (Lizcano, 2012).

Thomas Humphrey Marshall (1998) propone la ciudadanía como un estatus conducente a derechos y deberes en tres dimensiones: una civil, otra política y otra social, planteando la hipótesis de que la combinación de estos derechos apuntaría a la igualdad (Mata et al., 2013). La propuesta tripartita se transforma en un proceso concreto y simbólico, en tanto se nutre de significados, conceptos, relatos y discursos que sustentan la conducta y el imaginario cívico, determinando la participación de sus miembros. Sin embargo, el planteamiento canónico de Marshall (1998) ha sido discutido con posterioridad por diversos autores (Heater, 2007; Turner, 200I, citados por Mata et al., 2013), debido a que en la realidad no resulta suficiente para garantizar en forma efectiva los derechos plenos de todos sus miembros, pues legitima desigualdades y exclusiones en ámbitos de carácter étnico, de género, religioso, de clase, entre otros, y porque finalmente el concepto de "ciudadanía" se transforma en un título carente de contenido, es decir, se transforma en el ejercicio de una ciudadanía pasiva (Cortina, 1997). Es aquí donde se hace necesario que la sociedad pueda manejar de forma acertada esta convivencia multicultural, para lograr un sentido de pertenencia y adhesión de las personas a la comunidad, reconociendo a cada uno de sus integrantes de manera igualitaria y generando su decisión de participar libremente en proyectos colectivos. 
Acceder al campo de la ciudadanía requiere ubicarse primeramente en un contexto mundial contemporáneo que experimenta cambios vertiginosos, los cuales son posibles de apreciar a partir de la movilidad conductual de sus integrantes (Alejandre y Escobar, 2009). En este escenario global, los jóvenes se ubican en un segmento demográfico en tránsito y con múltiples rostros. Las generaciones de hoy consideran con mayor certeza que la vida es inestable, fluctuante, discontinua y sin una claridad en cuanto al momento en el cual son reconocidos como adultos. Al mismo tiempo, se aprecia la existencia de diferencias notables en su entramado, donde conviven diversas culturas juveniles asociadas a factores étnicos, de género, socioeconómicos, entre otros, que en definitiva dan cuenta de una construcción de vida diferente y distintiva entre ellos.

Desde la perspectiva del discurso ciudadano, la sociedad se ha esforzado por integrar a los jóvenes como sujetos aptos para participar en la vida pública, pero con restricciones asociadas a la construcción de competencias, habilidades y virtudes, junto con el permanente control ejercido a partir de la mirada adultocéntrica (Sandoval, 2003). El desafío se encuentra en lograr un impacto en las trayectorias de los jóvenes y superar las barreras impuestas entre lo estructural y lo cultural, esforzándose por entender las diversas expresiones contenidas en el discurso juvenil y avanzar hacia el fortalecimiento de programas que les garanticen derechos y beneficios reales, y no solo discursivos para su participación en la organización tradicional y electoral de la cual no se sienten parte.

Frente a los rápidos cambios y transformaciones de la sociedad actual, el informe Delors (1996) propone cuatro pilares para la educación del siglo XXI: aprender a conocer, aprender a hacer, aprender a ser y aprender a convivir. Destaca en particular este último aprendizaje, debido a que apunta hacia la comprensión de las otras personas, preparando a los educandos para una vida en comunidad, evitando los conflictos, relacionándose en un contexto de igualdad y tendiendo hacia objetivos comunes en clave pluralista. En esa misma línea, Cortina (1997) considera como valores fundamentales y propios de una ética cívica: la libertad, la igualdad, la solidaridad, el respeto activo y el diálogo. En cuanto a valores tales como la lealtad, la honradez, 
la profesionalidad, entre otros, considera que están articulados a los antes mencionados y que en su conjunto deben ser transmitidos mediante la educación.

Respecto de la formación ciudadana contextualizada en el modelo educativo chileno, (Flanagan, Cerda, Lagos y Riquelme, 2010) afirman que este es un proceso que aún se encuentra en desarrollo. En una revisión del acontecer en las últimas décadas se constata que la asignatura de "Formación Cívica", implementada en $3^{\text {er }}$ año medio entre I98I y I999, y que estaba centrada en conocimientos sobre el Estado y el sistema político, se reconvierte a fines de los años '90 a una "Formación Ciudadana", que incluye conocimientos, habilidades y actitudes que ya no están circunscritos a un nivel de enseñanza ni a una asignatura en particular, sino que son desarrollados de manera transversal, es decir, son promovidos a lo largo de toda la formación escolar en diferentes asignaturas (Miranda, 20I I).

\section{Contextualización social de la ciudadanía en Chile durante las últimas décadas}

El golpe de Estado ocurrido en Chile en septiembre de 1973 influyó en los actores sociales de la época, al punto que su legado cívico fue simplemente eliminado de la configuración social del país hasta I990, dadas las restricciones impuestas a la participación y el ejercicio de los derechos ciudadanos (Mártinez, Silva y Hernández, 2010). Con el término de la dictadura se generaron una serie de expectativas sobre el nuevo rol que ocuparía la sociedad civil; no obstante, los gobiernos de la Concertación optaron por una estrategia de priorizar la gobernabilidad del país por sobre las reivindicaciones sociales e históricas (Flanagan et al., 2010).

Sobre la base de esta decisión, la acción ciudadana se vio entonces dificultada por el escenario político que cuestionaba el verdadero Estado de Derecho, evitando sus componentes esenciales: el diálogo, la discusión y el debate, perdiéndose la perspectiva sobre la práctica democrática como un espacio de encuentro de comunidad, de país y de visiones de mundo (Flanagan et al., 2010). En esta misma línea de análisis, Miranda (20II) plantea que a este fenómeno 
contribuyeron también dos procesos claves: a nivel mundial, la globalización y la incorporación de las nuevas tecnologías al mundo moderno, que comenzaron a afectar de manera significativa las relaciones sociales cotidianas, produciendo de manera gradual un debilitamiento profundo en los vínculos sociales; a nivel local, el alejamiento de las personas de la vida social, que ha repercutido en una creciente disminución del grado de su involucramiento en las prácticas ciudadanas.

Toda esta situación fue levantando un progresivo desinterés en los jóvenes por participar de los circuitos formales de la política, así como de las acciones ciudadanas tradicionales; con ello, la sociedad civil en su conjunto consolida la idea de que su participación ciudadana poco puede influir en la construcción y operación de un proyecto país (Flanagan et al., 2010). Esta realidad no es exclusiva de Chile y así lo manifiestan Cabrera, Marín, Rodríguez y Espín (2005) en un estudio realizado a jóvenes españoles, en el cual la tendencia los sitúa en un estado pasivo en términos de derechos y deberes ciudadanos. Reconocen sus facultades en torno a la participación cívica, pero mantienen muy poca predisposición para ejercerlas en la medida en que no asimilan el deber recíproco que implica el derecho a participar. Junto con ello, y dada la realidad geográfica de la Península, surge el concepto de ciudadanía multicultural (Cabrera et al., 2005), con una actitud poco receptiva hacia la diversidad cultural de la sociedad, asociando a ciertos grupos económicamente desfavorecidos (ya sea inmigrantes $u$ otras culturas muy distintas) con situaciones de delincuencia y conflictos de convivencia. El contexto sudamericano también se asemeja a la experiencia chilena, puesto que países como Argentina y Brasil también evidencian problemas en la construcción de ciudadanía, que son antecedidos por regímenes autoritarios en los que la inclusión de estos temas dentro del currículo escolar involucra debates respecto de visiones de mundo e ideologías relacionadas con la ciudadanía como práctica y como realidad histórica (Mataluna, 20I I).

\section{Las representaciones sociales}

Al momento de abordar el estudio del concepto "ciudadanía", la tendencia es hacia los factores más inmediatos relacionados con la 
definición de los derechos de las personas ante grupos sociales y el Estado, transformándose en un objeto discursivo (Martínez, 2003). Sin embargo, la realidad muestra que la ciudadanía surge además como una construcción social, no como una realidad tangible, preexistente y estática, sino como algo que se va levantando desde la convivencia social (Gutiérrez, 20II), haciendo "posible la producción de vivencias dotadas de significación y sentido compartido que proveen de certezas al mundo de la vida de los ciudadanos" (Martínez, 2003, p. 2). Desde esa perspectiva, es necesario contar con una postura teórico-metodológica que permita el abordaje de la construcción social de los sujetos, siendo la teoría de las representaciones sociales aquella que permitirá conocer el sistema lógico de pensamiento social que poseen los miembros de un grupo con una determinada identidad (Arango, 2008).

Según Jodelet (20I I), las representaciones sociales corresponden a una forma específica de conocimiento, construido de manera social y compartido al interior de diferentes grupos que tiene una base y un objetivo práctico relacionado con el establecimiento de una guía u orientación para el comportamiento de las personas en la vida práctica y cotidiana. Esta teoría tiene su punto de partida en el campo de la psicología como ciencia experimental por el pensador alemán Wundt, quien, a través de sus estudios, distinguió entre la psicología experimental y la psicología social, buscando una forma de comprensión para fenómenos complejos en la vida del individuo relacionados con procesos cognitivos superiores del hombre y con la interpretación de los procesos de la experiencia colectiva, donde la "comunicación de gestos" asentaban las bases para la vida social, tales como el lenguaje y las costumbres (Mora, 2002). Posteriormente, Durkheim se interesó por las propuestas de Wundt y diferenciaba entre representaciones individuales y representaciones colectivas, en las que "la conciencia colectiva trasciende a los individuos como una fuerza coactiva y que puede ser visualizada en los mitos, la religión, las creencias y demás productos culturales colectivos” (Mora, 2002 p. 6).

Pero es Serge Moscovici quien, en décadas posteriores, retoma estos planteamientos para desarrollar una teoría etnopsicológica con una marcada tendencia sociológica, a la cual denomina "representaciones 
sociales", diferenciándose de las formas de concebir la psicología, que hasta ese momento se concentraba en lo individual (Mora, 2002). Entendía este concepto como "organizaciones paradigmáticas de creencias y conocimientos que comprenden aspectos individuales, sociales y culturales"; por lo tanto, "estas representaciones son simultáneamente individuales y sociales, puesto que son elaboradas por un sujeto que se encuentra en un intercambio comunicativo con otros y situado en un ambiente cultural específico”, en un proceso de comunicación mediatizado por el lenguaje, acción por la cual se constituye "en una forma de conocimiento socialmente elaborado" (Cisternas, 2012, p. 63).

En un nivel estructural, dentro de una representación social, Moscovici plantea que la información consensuada y más rígida corresponde al núcleo, siendo el responsable de la identidad de la representación, influenciada por condiciones de tipo histórico, social o económico del grupo donde se desarrolla, es decir, determinada por la memoria colectiva. Junto con ello, la que posee mayor grado de maleabilidad se denomina "periferia" y preserva a la representación social por su flexibilidad y adaptación (Pardo, 2007). En cuanto al surgimiento de una representación social, Moscovici señala dos procesos: la objetivación, entendida como la concreción de los conceptos que llevan a la construcción de imágenes que referencian al discurso, con la finalidad de hacer real un esquema conceptual, y el anclaje, que implica la integración del objeto de la representación al sistema preestablecido que posee creencias compartidas socialmente, siendo su característica fundamental el nombrar, entendida como un proceso de categorización (Pardo, 2007).

\section{Situación de la educación media técnico- profesional en Chile}

La educación media técnico profesional (en adelante EMTP) es una modalidad educativa que tiene por objetivo colaborar con el desarrollo de transiciones exitosas para sus estudiantes hacia el mundo laboral de manera temprana, mediante la obtención de las competencias que requiere un campo educativo o especialidad formativa en específico (Larrañaga, Cabezas y Dussaillant, 20I4). Su 
instalación se remonta a 1965, en el contexto de la reforma impulsada por el gobierno de Eduardo Frei Montalva, con una oferta secundaria de dos modalidades, una humanística-científica y otra técnicoprofesional. En la actualidad la EMTP contempla cuatro años de estudio, de los cuales los últimos dos están enfocados en responder a la demanda de mano de obra especializada, dado el contexto del campo laboral, al tiempo de eventualmente continuar con estudios superiores (Contador, 20II). Este segmento de la educación secundaria no ha estado ajeno a las consecuencias del golpe militar y su intervención en el mundo formativo, con las que el modelo de mercado instalado por la dictadura impactó en aspectos tales como la oferta de especialidades y su calidad y pertinencia respecto del mundo productivo y su vinculación con las escuelas, situación que no consideró una regulación en su crecimiento, así como tampoco la preparación y formación continua de sus docentes para afrontar dichas modificaciones curriculares.

En términos de importancia y pertinencia, la EMTP ocupa un lugar de relevancia para el desarrollo de las personas y la sociedad, situación que se manifiesta desde hace varios años en distintos países de América y de Europa (OCDE, 20I4). El impacto en Chile no es una excepción: el informe de encuesta Casen (Sepúlveda, 2008) señala que alrededor del 65\% de la matrícula EMTP corresponde a familias que provienen de los dos quintiles de menores ingresos, y supera el $80 \%$ si se agrega el tercer quintil de ingresos. Por su parte, Larrañaga, Cabezas y Dussaillant (2013) analizan un estudio realizado por el Programa de las Naciones Unidas para el Desarrollo (PNUD), en el que se concluye que quienes egresan de la EMTP acceden a trabajos mejores y con mayor remuneración respecto de aquellos estudiantes provenientes de la educación media científico humanista y, además, tienen la posibilidad de continuar sus estudios en un nivel superior. Lo anterior ubica a este tipo de formación como una alternativa concreta para los jóvenes de familias con más bajos ingresos, conformando este segmento el $4 \mathrm{I} \%$ de la matrícula total de la enseñanza media en Chile (Larrañaga, Cabezas y Dussaillant, 2013), cifra que no se ha modificado sustancialmente en relación a la década anterior, y que otorga un protagonismo evidente dentro del quehacer educativo del país. Si bien sus estudiantes tienen 
entre sus objetivos curriculares la inserción temprana en el mundo laboral y de manera eventual una posterior prosecución de estudios superiores, se espera que esto vaya acompañado de un proyecto de ciudadanía que les permita ser partícipes activos en los diversos lugares donde se inserten con posterioridad a su egreso del espacio formal en la escuela.

\section{Metodología}

Esta investigación está situada en el enfoque cualitativo, considerando que su objetivo es conocer las representaciones que los estudiantes de EMTP poseen sobre el concepto de "ciudadanía”. En este caso, la orientación hermenéutica permite acceder al conocimiento mediante el estudio de las construcciones discursivas para comprender su significado, tomando como base que no existe un saber objetivo, desinteresado y con un ser humano imparcial ante los sucesos del mundo (Osorio, 2007). Dicha teoría enfoca en el discurso como su fenómeno de interés, que surgirá desde las representaciones sociales de los estudiantes indagados, siendo estas últimas un ámbito de manifestación de la realidad a la que se puede acceder por medio del lenguaje como herramienta de interacción social (Moscovici, 1979).

El diseño responde a un estudio de caso (Pérez, I994) con alcance descriptivo (Hernández, Fernández y Baptista, 2010), debido a que se caracterizarán las representaciones de los sujetos respecto al concepto de "ciudadanía". El estudio es de naturaleza no experimental y transeccional (Hernández et al., 2010).

\section{Objetivo general}

Conocer las representaciones sociales de los estudiantes de EMTP sobre ciudadanía.

\section{Objetivos específicos}

- Identificar cuál(es) es (son) las representaciones sociales sobre ciudadanía que poseen los estudiantes de EMTP.

- Categorizar, a través del discurso, las representaciones sociales sobre ciudadanía de los estudiantes. 


\section{Participantes e instrumentos}

Los sujetos de estudio son 12 estudiantes de $4^{\circ}$ año de educación media pertenecientes a la formación diferenciada técnico-profesional de un establecimiento particular subvencionado de la ciudad de Santiago, administrado por la congregación salesiana, elegidos mediante una selección intencionada y no probabilística, a los cuales se les aplicó entrevistas semiestructuradas para recoger datos que expresaron a través del lenguaje.

\section{Análisis de la información}

Se realizó mediante análisis de contenido (Piñuel, 2002), para indagar en las subjetividades e identificar las estructuras de significado sobre las cuales se articulan las representaciones sociales en relación con el concepto de "ciudadanía" de los sujetos estudiados. Se levantaron categorías a priori (Cisterna, 2005), las cuales se formularon en relación a los objetivos planteados que se presentan en la siguiente tabla:

Tabla $\mathrm{N}^{\circ} \mathrm{I}$ : Categorías a priori generadas para la dimensión relacionada con ciudadanía. Fuente: Elaboración propia.

\begin{tabular}{|c|l|}
\hline DIMENSIÓN & \multicolumn{1}{c|}{ CATEGORÍAS } \\
\hline \multirow{3}{*}{ Ciudadanía } & Significados acerca del término "ciudadanía" \\
\cline { 2 - 2 } & Agentes que han formado el concepto de "ciudadanía" \\
\cline { 2 - 2 } & Condiciones requeridas para ser ciudadano \\
\cline { 2 - 2 } & Valores relacionados con ciudadanía \\
\hline
\end{tabular}

Dentro del análisis para cada categoría, se realizó una codificación previa para identificar al informante aludido al momento de efectuar la mención a la respuesta involucrada en el análisis del contenido vertido en la entrevista, denominando a cada entrevistado como "E", seguido de un número que alude al correlativo del encuentro al momento de su aplicación, esto es, estudiante o entrevistado I: EI; estudiante o entrevistado 2: E2, y así, sucesivamente, dentro de un total de doce participantes en el proceso. 


\section{Resultados}

\section{Significados sobre el término "ciudadanía"}

Para describir el significado del concepto de "ciudadanía" los estudiantes manifiestan variadas interpretaciones. Apelan inicialmente y de manera mayoritaria a un aspecto de carácter territorial relacionado con el sentido de pertenencia a un lugar o de personas que habitan en un entorno comunitario.

A lo que uno pertenece, uno puede pertenecer a una ciudadanía teniendooo..., dando sus opiniones también, y cosas así. Ese es mi..., un poco lo que tengo como referencia de lo que es ciudadanía. (E2)

Es como..., es como sentirse parte de aloo..., por ejemplo, yo ya soy ciudadano colombiano, porque me siento como parte de Colombia..., es como ese arraigo que tu tienes bacia un lugar... (E4)

Y ciudadano es vivir po', vivir en comunidad... en un país, una región... (ElO)

En segundo lugar, relacionan la ciudadanía con elementos de índole civil y política, en la que aparecen mencionados tanto la obtención de derechos como el cumplimiento de leyes y normas dentro de la sociedad.

Las votaciones... [...] Porque abí es donde uno ejerce la ciudadanía..., la bace válida..., comillas... (risas)..., de alguna manera..., con el voto abí en la urna..., el voto secreto $y . .$. , todas esas cosas..., porque es algo que se adquiere cuando un obtiene 18 años..., y abí uno dice ser ciudadano ya..., del..., del país. (E5)

Es como los derechos que tiene una persona al ser parte de un país, pero también es como la gente que babita el país, aunque más generalmente se le conoce como eso..., tú bablas de ciudadanía y dices: la ciudadanía es Santiago..., como la gente que vive en ciertos sectores... (E4)

Nosotros, como ciudadanos, debemos acatar leyes que las tiene el Estado en base a la Constitución, o sea, ondaaaa..., nosotros tenemos que obedecer la Constitución, o a las leyes que otorga el Estado y eso es como un derecho de ser ciudadano... (ElO)

También surge en el discurso de los entrevistados una mirada de carácter social frente al término "ciudadanía", vinculada con las 
expresiones de la gente, la posibilidad de opinar, de participar en diversas actividades comunitarias y la preocupación por el bienestar del otro.

Todo lo que es el entorno en la ciudad, las expresiones de la gente, etcétera... y que uno tiene el derecho de expresar algo, eso es ciudadanía..., estar unidos, valorar las cosas, dar expresiones, alegar sobre sus derechos..., eso es ciudadanía. (E8)

Para mí la ciudadanía es cuando unuunn..., una persona pasa de ser de quien se preocupa por sí mismo a una persona que en conjunto ayuda a la organización de algo, en este caso puede ser un país, un grupo pequeño..., y rasgos importantes para mí de esto es que tiene que siempre estar a favor de las personas y no en su contra. [...] Además, cuando pienso en ciudadanía se me viene como la idea de una agrupación también..., donde la gente es empática y trabaja de manera conjunta... (E9)

\section{Agentes que han formado el concepto de "ciudadanía"}

Dentro de la construcción de significados en torno al concepto de "ciudadanía", los entrevistados manifiestan a la familia como el primer referente de aprendizaje, principalmente los padres, a través de las conversaciones sobre temas de actualidad en los momentos de encuentro y convivencia al interior de este núcleo.

Primero bay una visión de familia, yo creo también que..., en sí es bien política, en mi familia se babla mucbo de política, bien centrado al izquierdismo chileno... (E6)

Mis padres igual me bablan bastante de..., de lo que pasa en el entorno, que bay que tener cuidado con los robo'..., más mi madre me babla sobre el tema... (E8)

Mis mismos padres, familiares cercanos, tíos, parientes, bermana, que ban podido de alguna manera con otras visiones de ella..., ellos como agentes que tengo, como de formación más cercano... (El l)

También aparece mencionado el colegio como un agente de relevancia en este proceso de aprendizaje sobre ciudadanía, tanto desde una mirada global como particularizada en los profesores que dictan la asignatura de Historia, Geografía y Ciencias Sociales. 
Los profesores, los profesores, eeeeebbhb..., también el entorno social, como están todos..., eeeeebbhb..., política también, eeeeebhbh..., pero más los profesores, de Historia, que nos están incluso pasando lo que es ciudadanía. (E2)

También el colegio te enseña, te enseña mucbo yo creo..., bueno, los profesores de Historia se enfocan en enseñarte los conceptos y todo..., o sea es la base de todo, la educación que se forma en el colegio y en la casa. (E6)

Los que me ban bablado más ban sido profesores, que..., el que me ba bablado más de esto fue un profesor de octavo que se llama Mauricio... (E7)

Junto con ello, surgen algunas manifestaciones en relación con el uso de internet como herramienta el aprendizaje y la construcción de estos significados. Se destaca tanto el espacio de la red global como los grupos de reunión y discusión virtual.

También la..., no sé si podrá ser, pero..., en internet también bay mucba cosa que se puede leer y que de alguna manera influye..., no tanto como lo bace directamente la familia o los amigos, pero..., que uno queda abí pensando y reflexionando... (E5)

Ab, y también con personas lejanas, o sea, en línea..., en los foros de software libre, por ejemplo, que abí se encuentran distintas mentalidades, porque todos son de distintos países..., la gente actúa diferente, entrega ayuda de manera diferente, $y$ de abí be ido sacando mis propias conclusiones y ba influido en mis ideales, por decirlo de alguna manera. (E9)

\section{Condiciones requeridas para ser ciudadano}

Frente a las condiciones requeridas para ser ciudadano, los estudiantes manifiestan algunas visiones dispares, orientadas por una parte a los aspectos formales de la civilidad, tales como estar inscrito en el Registro Civil, la posesión de la nacionalidad y el cumplimiento de la mayoría de edad, entre otros; por otro lado, se menciona el rol político que debe cumplir un ciudadano, mediante el respeto y cumplimiento de las leyes y de las normas establecidas en la Constitución.

Ser nacido..., en Chile por decirlo así.., eeeeebhbh..., registrarte como ciudadano, ir como al Registro civil y ya, registrarte que soi' ciudadano..., eeeeebbbh..., 
tener un nombre más que nada, que eso va ya como en cuando uno se registra, que dice ya, soy ciudadano, cuando te ponen un nombre, te dan un RUT, y yo creo que con eso ya basta para ser ciudadano... (E3)

Cumplir los 18 años, y tener la capacidad de poder elegir..., de poder pensar y después elegir al candidato..., al que uno estima que es bueno o que alguna propuesta..., dentro de lo posible es buena... (E5)

Respetar las leyes que establece la Constitución, eeeeebbbh..., y como se llama, ser libre en el aspecto del qué decir y que esa opinión valga en cuanto al Estado..., se puede apreciar en las elecciones presidenciales, de concejales y toda' esas cosas... (ElO)

Junto con ello, algunos jóvenes plantean una mirada desde una dimensión más bien social, en la que la posibilidad de opinar, el sentido de pertenencia a un lugar o territorio y la búsqueda del bien común se transforman en piezas claves para ser y sentirse ciudadano.

Yo creo que generalmente uno como..., es más interior..., como que no necesita que una persona lo reconozca como tal, sino que uno se siente más arraigado al terreno... (E4)

Para que una persona sea reconocida como ciudadano, yo creo que tiene que ser una persona que tenga una opinión frente a los temas de política que tiene un país..., y tener la madurez suficiente para poder dar esa opinión... (E6)

O sea, para ser un ciudadano es más que nada demostrar la voluntad de ayudar a otras personas, de ser parte de un conjunto de ayuda mutua..., baciendo méritos o labores que siempre busquen estar por sobre..., el bien personal, para mejorar la calidad de vida de aquellos que los rodean..., con eso bastaría para mí. (E9)

\section{Valores relacionados con ciudadanía}

Frente al tema valórico, la responsabilidad, el respeto y la solidaridad se consolidan entre los estudiantes como aquellos valores relacionados con el ejercicio de la ciudadanía, para la defensa de las ideas y propuestas que la persona tenga, la tolerancia por las posturas diferentes dentro de la sociedad y la ayuda y colaboración hacia los demás dentro de un espacio de convivencia en común. 
Principalmente yo creo que con el valor de la responsabilidad, ya que bay que tener un compromiso claro con todo lo que uno plantea y todo lo que uno defiende como ideales... (E6)

O sea, más que nada con el respeto mutuo bacia los demás, eso es como lo fundamental en todo ámbito, siempre el respeto..., yo soy ciudadano, respeto ciertas normas, ciertas leyes y no trato de sobrepasar a los demás de mi entorno, para mí el respeto es como lo más fundamental... (EI l)

También ellos mencionan otros valores importantes para un buen ejercicio ciudadano, tales como la empatía, el compromiso, la honestidad, la honradez, entre otros, situación que refrenda el planteamiento de Cortina (1997), en tanto existen valores primordiales que engloban a otros y que en su conjunto pueden considerarse como universalmente humanizadores.

Responsabilidad, consistencia y empatía por las personas, así como para ayudar a los demás... (E9)

Ser bonesto, no sé po'..., bonesto porque con eso se puede así como..., pucba, bacer las cosas bien y no equivocarse..., solidario también, ante todo..., pa' ayudar a los más necesitados, yyyyyyyy..., como se llama..., no ser solamente uno el que tiene y el otro quede abí como, pucha, me falta algo con que me ayuden... (ElO)

\section{Conclusión}

Los significados fundamentales que manifiestan los estudiantes sobre "ciudadanía" se relacionan con un primer elemento de índole territorial, es decir, de sentido de pertenencia a un lugar o de un grupo de personas que habitan y son parte de una determinada comunidad. En segundo término, surge la conexión con aspectos de índole civil y política, en los que aparecen mencionados tanto la obtención de derechos como el cumplimiento de leyes y normas dentro de la sociedad. En tercer lugar, aparece el carácter social del término, vinculado a las expresiones de la gente, la posibilidad de opinar, la participación en diversas actividades comunitarias y la preocupación por el bienestar del otro. 
En cuanto a la definición de agentes que los estudiantes consideran como aportes para la construcción de estos significados, hay un amplio nivel de consenso respecto de visualizar a la familia como el primer referente de aprendizaje, principalmente los padres. En segunda instancia emerge la escuela como institución que también entrega conocimientos sobre esta temática, tanto desde una perspectiva de conjunto como en el abordaje efectuado en la asignatura de Historia, Geografía y Ciencias Sociales. El uso de la red global de información, internet, también aparece identificada como una herramienta para la exploración de este campo, así como para la interacción con otras personas de manera virtual.

Las condiciones requeridas para ser reconocido como ciudadano presentan una visión de disparidad entre los entrevistados, con manifestaciones en torno a los aspectos formales de la civilidad tales como la inscripción en el Registro Civil, la posesión de la nacionalidad y el cumplimiento de la mayoría de edad, entre otros; se menciona además el rol político, a través del respeto y cumplimiento de las leyes y normas establecidas por la Constitución. También surge una mirada más bien social, en la que el derecho a opinión, el sentido de pertenencia a un lugar o territorio y la búsqueda del bien común se transforman en elementos esenciales para ser y sentirse ciudadano.

Frente a los valores relacionados con el ejercicio de la ciudadanía, aquellos que destacan en la construcción de significados para los jóvenes son la responsabilidad, el respeto y la solidaridad, ya que permitirían la promoción y defensa de ideas, la tolerancia ante posturas disímiles y la ayuda y colaboración hacia los demás en un espacio de convivencia común. Junto a los primeros se mencionan la empatía, el compromiso, la honestidad, la honradez, la lealtad, el trabajo, entre otros. Estos valores se entienden como elementos universalmente humanizadores, donde los segundos se encuentran insertos en algunos de los primeros mencionados al momento de su operacionalización. Llama la atención que, frente a los valores fundamentales y propios de una ética cívica, no se mencione en los discursos de los jóvenes la libertad y la igualdad, a pesar que el primero ha estado en permanente discusión a nivel nacional producto del régimen vivido luego del golpe de Estado de 1973; el segundo 
ha formado parte de las movilizaciones y reivindicaciones por las cuales las nuevas generaciones de estudiantes han venido luchando desde 2006.

Todo lo anterior da cuenta, en su globalidad, de un conocimiento altamente heterogéneo respecto al concepto de "ciudadanía" por parte de los estudiantes, situación que implicaría la elaboración y posterior implementación un plan de trabajo concertado desde toda la comunidad educativa que conforma a la escuela, y que se oriente al abordaje de esta temática con una mirada proyectiva y de conjunto, que complemente y/o fortalezca las visiones ya instaladas en los estudiantes desde el seno familiar, en el entendido que son estas dos instituciones las reconocidas por ellos como principales agentes de contribución en la construcción de significados sobre este tema.

Bajo una mirada reflexiva, resulta pertinente colaborar desde los espacios educativos con la recomposición de la sociedad chilena, atropellada sistemáticamente en sus derechos individuales y colectivos durante la dictadura militar, y cuyo tejido social no fue rearticulado por los siguientes gobiernos ante decisiones de carácter político. Se requiere entonces contar con jóvenes preparados conceptualmente, educados en la libertad, con capacidad crítica y comprometidos con el ejercicio ciudadano en sus múltiples esferas, situación que puede entregar garantías de avanzar cada vez más en la defensa de los derechos de todas la personas y del sistema democrático nacional en su conjunto, potenciando al mismo tiempo los sentidos de pertenencia e identidad, claves para hacerse parte real de la sociedad en la cual se insertarán y desarrollarán.

En cuanto a las proyecciones investigativas que surgen a partir de los resultados expuestos, se observa la necesidad de analizar en profundidad los niveles educativos iniciales dentro de la enseñanza media, con el fin de recabar información respecto de cómo efectivamente los jóvenes en esas edades tempranas conciben el concepto de "ciudadanía", quiénes son o han sido sus informantes y cuáles los espacios de participación donde se desenvuelven en este proceso de adolescencia, siempre cargado de influjos e información provenientes de todos los ámbitos de sus vidas. 


\section{Referencias bibliográficas}

Alejandre, G., y Escobar, C. (2009). Jóvenes, ciudadanía y participación política en México. Espacios Públicos, 12(25), I03-I22.

Arango, L. (2008). Representaciones y prácticas sobre ciudadanía en estudiantes de octavo grado de educación básica secundaria en tres planteles educativos pertenecientes a estratos sociales diferentes. Medellín, Colombia: Universidad de Antioquia.

Asún, R., Ruiz, S., Aceituno, R., Venegas, J. y Reinoso, A. (2012). Integración subjetiva y apoyo institucional percibido entre estudiantes de secundaria en Chile. Revista Latinoamericana de Ciencias Sociales, Niñez y Juventud, IO(I), 32I-338.

Berger, P., y Luckmann, T. (200I). La construcción social de la realidad. Buenos Aires: Amorrortu Editores.

Cabrera, F., Marín, M., Rodríguez, M. y Espín, J. (2005). La juventud ante la ciudadanía. Revista de Investigación Educativa, 23(I), I33-I72.

Cisterna, F. (2005). Categorización y triangulación como procesos de validación del conocimiento en investigación cualitativa. Theoria, 24(I), 6I-7I.

Cisternas, L. (2012). Las representaciones de ciudadanía en jóvenes secundarios cbilenos: construcción de ciudadanía juvenil. Santiago, Chile: Universidad de Chile.

Contador, A. (20II). La formación diferenciada técnico profesional: una mirada a través de las voces de docentes de especialidades, en un liceo municipal. Santiago, Chile: Universidad de Chile.

Cortina, A. (1997). Cuidadanos del mundo: bacia una teoría de la ciudadanía. Madrid: Alianza Editorial .

Delors, J. (I996). La educación encierra un tesoro. Madrid: Santillana.

Flanagan, A., Cerda, G., Lagos, D. y Riquelme, S. (2010). Tensiones y distensiones en torno a la ciudadanía y formación ciudadana: Comparación de los significados de los profesores y estudiantes secundarios en la región de Valparaíso. Última década, l8(33), I I5-I37.

Gutiérrez, S. (20II). Representaciones sociales y construcción de la ciudadanía en jóvenes universitarios. Sinéctica, 36, I-I8.

Hernández, R., Fernández, C. y Baptista, P. (2010). Metodología de la Investigación. México: Mcgraw-Hill.

Jodelet, D. (20II). Aportes del enfoque de las representaciones sociales al campo de la educación. Especios en blanco. Serie Indagaciones, 2 l(I), I33-I54.

Larrañaga, O., Cabezas, G., y Dussaillant, F. (20I4). Estudio de la educación técnico profesional. Estudios Públicos, (I34), 7-58. 
Larrañaga, O., Cabezas, G. y Dussaillant, F. (2013). Estudio de la educación técnico profesional. Santiago, Chile: PNUD.

Lizcano, F. (2012). Conceptos de ciudadano, ciudadanía y civismo. POLIS, ll(32), 269-304.

Marshall, T. (1998). Ciudadanía y clase social . Madrid: Alianza .

Mártinez, G. (2003). Internet y ciudadanía global: procesos de producción de representaciones sociales de ciudadanía en tiempos de globalización. Aposta Revista de Ciencias Sociales, (9), I-20.

Mártinez, M., Silva, C., y Hernández, A. (2010). ¿En que ciudadanía creen los jóvenes?: Creencias, aspiraciones de ciudadanía y motivaciones para la participación sociopolítica. Psykhe, $19(2)$.

Mata, P., Ballesteros, B. y Padilla, M. (2013). Ciudadanía participativa y transformadora: análisis de discursos y propuestas de aprendizaje. Teoría Educación, 25(2), 49-68.

Mataluna, M. (20II). Escolaridad y ciudadanía: un estudio comparado de Argentina y Brasil. PROLAM/UPS: VI Jornadas de Jóvenes Investigadores.

Miranda, V. (20II). Competencias ciudadanas en estudiantes secundarios. Revista de Psicología, l(I), 34-5I.

Mora, M. (2002). La teoría de las representaciones sociales de Serge Moscovici. Athenea Digital, 2, I-25.

Moscovici, S. (1979). El Psicoanálisis, su imagen y su mundo. Buenos Aires: Huemul.

OCDE. (2014). Panorama de la educación. Indicadores OCDE. Santiago, Chile: Santillana.

Osorio, F. (2007). Epistemología de las ciencias sociales. Breve manual. Santiago, Chile: Ediciones UCSH.

Pardo, N. (2007). Cómo bacer análisis crítico del discurso: una perspectiva latinoamericana. Santiago, Chile: Frasis.

Pérez, G. (1994). Investigación Cualitativa I: Retos e interrogantes (6º edición). Madrid: La Muralla.

Piñuel, J. (2002). Epistemología, metodología y técnicas de análisis de contenido. Estudios de sociolingüistica, 3(I), I-42.

Sandoval, J. (2003). Ciudadanía y juventud: el dielma entre la integración social y la diversidad cultural. Última década, 19, 25-31.

Sepúlveda, L. (2008). La enseñanza media técnico profesional en Chile: Orientaciones actuales desde la perspectiva de sus actores. En Evidencias 
Representaciones sociales sobre ciudadanía en estudiantes de educación media técnico profesional - Toro

para políticas públicas en educación. Selección de investigaciones Tercer concurso FONIDE (pp. 15-52). Santiago, Chile: Centro de Estudios MINEDUC. 